Published in final edited form as:

Arch Sex Behav. 2015 October ; 44(7): 1861-1867. doi:10.1007/s10508-015-0505-5.

\title{
Pilot Randomized Controlled Trial of an Integrative Intervention with Methamphetamine-Using Men Who Have Sex with Men
}

\author{
Adam W. Carrico, \\ University of California San Francisco School of Nursing, 2 Koret Way, N511M, San Francisco, \\ CA 94143, USA \\ San Francisco Center for AIDS Prevention Studies, University of California, San Francisco, CA, \\ USA \\ Walter Gómez, \\ University of California San Francisco School of Nursing, 2 Koret Way, N511M, San Francisco, \\ CA 94143 , USA \\ Michael D. Siever, \\ San Francisco AIDS Foundation, San Francisco, CA, USA \\ Michael V. Discepola, \\ San Francisco AIDS Foundation, San Francisco, CA, USA \\ Samantha E. Dilworth, and \\ San Francisco Center for AIDS Prevention Studies, University of California, San Francisco, CA, \\ USA \\ Judith T. Moskowitz \\ Department of Medical Social Sciences, Northwestern University, Chicago, IL, USA \\ Adam W. Carrico: adam.carrico@ucsf.edu
}

\section{Abstract}

Contingency management (CM) is an evidence-based intervention that provides tangible rewards as positive reinforcement for biologically confirmed abstinence from substance use. Integrative approaches targeting positive affect regulation could boost the effectiveness of CM by sensitizing individuals to non-drug-related sources of reward and assisting them with effectively managing symptoms of withdrawal. This pilot randomized controlled trial with 21 methamphetamine-using men who have sex with men (MSM) examined the feasibility and accept-abilityofa5-session, positive affect intervention delivered during CM-Affect Regulation Treatment to Enhance Methamphetamine Intervention Success (ARTEMIS). After completing 4 weeks of a 12-week CM program, participants were randomized to receive ARTEMIS+CM $(n=12)$ or CM-only $(n=9)$. Those randomized to receive the ARTEMIS positive affect intervention completed $98 \%$ of sessions and reported marginally significant increases in positive affect over the five sessions. In exit interviews with ARTEMIS+CM participants, individuals noted that the positive affect regulation skills increased self-awareness and led to greater engagement in the recovery process. 
ARTEMIS+CM participants reported significant increases in positive affect and CM-only participants reported significant reductions in negative affect over a 2-month follow-up. These affective changes were not maintained, and no concurrent effects on stimulant use or sexual risk taking were observed over the 6-month follow-up. More definitive clinical research is necessary to ex-amine the efficacy of ARTEMIS+CM with methamphetamine-using MSM.

\section{Keywords}

Contingency management; Men who have sex with men; Methamphetamine; HIV/AIDS; Sexual orientation

\section{Introduction}

Among men who have sex with men(MSM), there is emerging evidence of a syndemic multiple, overlapping psychosocial health problems that fuel the HIV/AIDS epidemic (Mustanski, Garofalo, Herrick, \& Donenberg, 2007; Parsons, Grov, \& Golub, 2012; Stall et al., 2003). Informed by Syndemics Theory (Stall, Friedman, \& Catania, 2008), the experience of social adversity is theorized to potentiate the development of these cooccurring psychosocial health problems including stimulant use, depression, sexual compulsivity, and intimate partner violence (Herrick et al., 2013). Although the cooccurrenc of syndemic conditions has important implications for HIV/AIDS prevention, stimulant use is independently associated with increased risk for HIV seroconversion among MSM (Koblin et al., 2006; Ostrow et al., 2009). HIV-positive, stimulant-using MSM are also more likely to report engagingin HIV transmission risk behavior and display elevated HIV viralload, which together increase risk for onward HIV transmission (Carrico et al., 2011; Johnson, Carrico, Chesney, \& Morin, 2008; Mayer, Skeer, O'Cleirigh, Goshe, \& Safren, 2014). Taken together, innovative interventions targeting stimulant use have the potential to optimize HIV/AIDS prevention efforts among MSM.

Contingency management $(\mathrm{CM})$ is an evidence-based, behavioral intervention where individuals receive escalating tangible reinforcement for biologically confirmed drug abstinence (Prendergast, Podus, Finney, Greenwell, \& Roll, 2006). Prior research conducted with substance-using MSM has provided some evidence for the efficacy of CM as a standalone intervention. One randomized controlled trial (RCT) with homeless, substancedependent MSM observed a buffering effect of CM over a 12-month follow-up (Reback et al., 2010). Among those randomized to $\mathrm{CM}$, the proportion of urine samples that were reactive for cocaine, amphetamine, and methamphetamine remained relatively stable over the 12 months. In contrast, control participants who received HIV prevention programming activities displayed substantial increases in the proportion of urine samples that were reactive for these stimulants. These findings lend support to a prior RCT with methamphetamine-dependent MSM where those receiving $\mathrm{CM}$ alone or $\mathrm{CM}$ in combination with cognitive-behavioral therapy (CBT) were retained longer in treatment and had more consecutive urine samples that were negative for methamphetamine compared to men in CBT alone (Shoptaw et al., 2005). These initial benefits of CM observed during treatment dissipated over a 12-month follow-up such that all behavioral treatment conditions displayed 
sustained reductions in methamphetamine use and sexual risk taking. Further research is needed to develop and test novel strategies to boost and extend the effectiveness of CM with methamphetamine-using MSM.

A recent pilot RCT also highlights the potential pitfalls in effectively implementing CM with methamphetamine-using MSM (Menza et al., 2010). Due to poor participant attendance of $\mathrm{CM}$ visits, the $\mathrm{CM}$ protocol was modified to collect urine samples twice weekly instead of thrice weekly. Because this provides a window for methamphetamine use to go undetected, contingent reinforcement of stimulant abstinence was subverted. This may partially explain why men receiving CM displayed increases in methamphetamine use over a 6-month follow-up compared to those in an assessment-only control condition. Integrative intervention approaches could assist participants with effectively engaging in thrice weekly urine screening during $\mathrm{CM}$.

Resilience encompasses the social and psychological resources that assist MSM in effectively coping with social adversity (Herrick, Stall, Goldhammer, Egan, \& Mayer, 2014). Consistent with a compensatory model, resilience could directly ameliorate the burden of syndemic conditions and support HIV/AIDS prevention efforts in MSM. In particular, positive affect has been theorized to be an important source of psychological resilience that reinvigorates coping efforts in the midst of chronic stress and builds supportive social networks that assist individuals with managing stressful circumstances (Folkman \& Moskowitz, 2000; Fredrickson, 1998). Lending support to its adaptive significance, positive affect is associated with reduced sexual risk taking behavior in MSM and enhanced HIV disease management (Carrico, Johnson, Colfax, \& Moskowitz, 2010; Carrico \& Moskowitz, 2014; Mustanski, 2007). Findings from a recent cross-sectional study with methamphetamine-using MSM also indicate that positive affect is indirectly associated with decreased stimulant use via enhanced cognitive-behavioral change processes and greater abstinence-specific social support (Carrico et al., 2013). An intervention designed to increase positive affect could sensitize individuals to non-drug-related sources of reward and assist with managing symptoms of withdrawal (e.g., depression) that contribute to stimulant relapse (Carrico, 2014).

This pilot RCT examined the feasibility and acceptability of a positive affect intervention designed to serve as an adjuvant to CM with methamphetamine-using MSM. Participants were enrolled in a 12-week, community-based CM program in San Francisco that is being implemented as one component of a broader public health response to the intertwined epidemics of methamphetamine use and HIV AIDS (Carrico et al., 2014; Shoptaw et al., 2006; Strona et al., 2006). Feasibility was examined by determining the degree to which participants engaged in a positive affect intervention delivered concurrently with CM. Acceptability was assessed using exit interviews to characterize the experiences of participants with the positive affect intervention. Finally, preliminary outcomes of this integrative intervention approach were examined over a 6-month follow-up period. 


\section{Method}

\section{Participants and Procedure}

Participants were recruited from a community-based, 12-week CM program for methamphetamine-using MSM. All participants completed a run-in period such that they were invited to be screened only after engaging in 4 weeks of CM. At screening, participants were required to meet the following eligibility criteria: (1) identify as male; (2) report having anal sex with a man in the past year; and (3) report using methamphetamine at least weekly on average during the 3 months prior to $\mathrm{CM}$. All of the 21 individuals screened were eligible and willing to participate in a pilot RCT of a positive affect intervention-Affect Regulation Treatment to Enhance Methamphetamine Intervention Success (ARTEMIS). All participants completed aninitial study visit that included informed consent, and study procedures were approved by the Committee on Human Research at the University of California, San Francisco.

After completing a baseline assessment, participants were randomized to receive either a 5session, individually delivered ARTEMIS positive affect intervention during the remaining 8 weeks of CM (i.e., ARTEMIS+CM) or CM-only. Randomization was accomplished using a computer-based algorithm with randomly permuted block sizes. The randomization algorithm was only accessible to the study data manager. Follow-up assessments were conducted at 2-month post-randomization (i.e., immediately following the conclusion of $\mathrm{CM})$ as well as 3- and 6-month post-randomization. All study assessments were administered via computer. Measures of self-reported substance use and sexual risk taking behavior were completed by participants using audio computer-assisted self-interviewing to enhance reliability and validity (Des Jarlais et al., 1999). A urine sample was collected at each assessment for on-site toxicology screening. Participants received a $\$ 50$ pre-loaded debit card for completing each study assessment.

CM Program-Methamphetamine-using MSM enrolled in a community-based CM program completed urine screens thrice weekly and received escalating monetary reinforcement for samples that were non-reactive for methamphetamine. All participants were encouraged to pursue abstinence from methamphetamine and other stimulants during the 12-week CM protocol. Urine sample collection was directly observed by staff. The voucher for the initial methamphetamine-free sample was worth $\$ 2.00$. Vouchers increased in value by 25 cents for each consecutive methamphetamine-free sample to a maximum of $\$ 10.00$. Participants earned an $\$ 8.50$ bonus voucher for every third consecutive methamphetamine-free sample. A rapid reset procedure allowed participants to return to their place in the escalating reinforcement schedule after producing three consecutive urine samples that were non-reactive for methamphetamine. The total possible reinforcement was $\$ 330$, and participants could choose to receive incentives earned any time during or after the 12-week CM intervention period.

Concurrent with the CM urine screening visits, individuals could participate in a drop-in group that provided social support for continuing to pursue goals for stimulant use reduction or abstinence. Drop-in groups also provided opportunities to facilitate linkage to community-based services including formal substance abuse treatment. After completing the 
12 weeks of urine screening visits, individuals were invited to attend aftercare sessions to continue to forge social networks that are supportive of avoiding methamphetamine use and develop relapse prevention plans. Those who completed the 12-week CM program with aftercare were invited to continue attending regular drop-in groups to provide support and mentorship to men who were actively completing the thrice weekly urine screening visits.

ARTEMIS Intervention-ARTEMIS is a multi-component, individually delivered, fivesession intervention targeting positive affect that was adapted from prior clinical research testing the feasibility and acceptability of a positive affect intervention for recently diagnosed HIV-positive persons (Moskowitz et al., 2012). The original positive affect intervention protocol consists of eight distinct skills: (1) noticing positive events; (2) capitalizing on positive events; (3) gratitude; (4) informal and formal mindfulness; (5) positive reappraisal; (6) personal strengths; (7) attainable goals; and (8) acts of kindness. As shown in Table 1, adaptationsincluded additional content to optimize delivery of the core positive affect regulation skills with this population and new components designed to facilitate greater engagement in the recovery process (e.g., problem-focused coping skills training). Participants were asked to complete work sheets between sessions to facilitate home practice of the positive affect skills.

Informed by prior research examining the efficacy of mindfulness-based relapse prevention (Bowen et al., 2009, 2014; Witkiewitz, Bowen, Douglas, \& Hsu, 2013), participants also completed meditation exercises during ARTEMIS intervention sessions to further enhance metacognitive awareness and assist individuals in coping more effectively with methamphetamine withdrawal. Meditation exercises were pre-loaded on an iPod shuffle that was given to participants as an incentive to facilitate regular home practice. In exchange for their time and travel expenses, participants received $\$ 20$ cash for attending each intervention session.

\section{Measures}

Positive and Negative Affect-An adapted version of the Differential Emotions Scale (DES) assessed the frequency of positive and negative affect (Izard, 1977). The DES was modified to include more items assessing positive affect (Fredrickson, Tugade, Waugh, \& Larkin, 2003). Reliability for the 13-item positive affect (Cronbach's $\alpha=0.90)$ and the 11item negative affect (Cronbach's $a=0.84$ ) subscales of the DES was acceptable.

Stimulant Use-Participants reported the number of days they used methamphetamine in the past 30 days. Participants also provided a urine sample for on-site toxicology testing (Redicup®; Redwood Toxicology Laboratory, Inc.). Samples that were reactive for methamphetamine or cocaine metabolites (1) were compared to those that were not reactive for these stimulants (0).

Sexual Risk Taking Behavior-Participants reported the number of anal sex partners in the past 3 months, stratified by whether or not they were feeling the effects of methamphetamine during sexual intercourse. For HIV-negative MSM, sexual risk taking behavior was operationalized as engaging in unprotected anal intercourse, irrespective of the 
serostatus of the sexual partners. For HIV-positive MSM, sexual risk taking behavior was operationalized as unprotected anal intercourse with HIV-negative or unknown serostatus partners.

Exit Interviews-Immediatelyafterthe2-monthfollow-upassessment, participants randomized to receive ARTEMIS completed an exit interview to examine their experiences with the intervention. During the exit intervention, participants indicated on Likert-type scale from 0 (Not at All) to 10 (Definitely) how likely they would be to recommend the ARTEMIS intervention to a friend who uses methamphetamine.

\section{Data Analysis}

Quantitative analyses of data from the pilot RCT were conducted to examine the preliminary outcomes of ARTEMIS+CM. Among those randomized to receive the ARTEMIS intervention, changes in positive and negative affect were examined over the five sessions. Inferential analyses were also conducted to examine unadjusted change over the 6-month follow-up period for each dependent variable, stratified by intervention condition. Analyses were performed with generalized estimating quations (GEE) in SAS 9.3 using the binomial distribution and logit link for the proportion of urine samples that were positive for stimulants and the normal distribution and identity link for all other continuous dependent variables. GEE has been shown to be superior to traditional approaches to analyze repeated measures data (e.g., repeated measures ANOVA), particularly with small samples (Ma, Mazumdar, \& Memtsoudis, 2012). All tests of statistical significance were two-tailed ( $a<$ 0.05).Using qualitative data from exit interviews, sample descriptions of the experiences of participants with the ARTEMIS intervention were selected by two members of our team (i.e., AWC and WG) to provide context for quantitative ratings of satisfaction. Because exit interviews were conducted solely for evaluation purposes (Thomas, 2006), formal qualitative analyses (e.g., thematic analyses) were not conducted.

\section{Results}

\section{Enrollment and Follow-up}

Participants were enrolled between January 2011 and April 2011. Of the 21 participants randomized, 19 (91\%) completed at least one assessment during the 6 months after randomization. Of the two participants lost to follow-up, one ARTEMIS+CM participant was inaccessible because he enrolled in inpatient substance abuse treatment, and one CMonly participant was incarcerated. Follow-up rates at 2-, 3-, and 6-month post-randomization were $17(81 \%), 19(91 \%)$, and $18(86 \%)$ respectively.

\section{Demographics and Health Status}

The majority of participants were middle-aged $(M=41.1, \mathrm{SD}=9.0)$ men who described their sexual orientation as predominantly or exclusively gay $(n=15)$. The sample was multiethnic: 10 Caucasian, 5 African American, 5 Hispanic/Latino, and 1 of multi-cultural heritage. Half of the participants were well educated with 12completingatleast some college, 3 were employed at least part time, and 10 reported being homeless in the past year. Half ( $n$ = 11) of participants were HIV negative. The $10 \mathrm{HIV}$-positive participants had been living 
with HIV/AIDSforanaverageof16.1 ( $\mathrm{SD}=8.3)$ years and most $(n=9)$ were prescribed antiretroviral medications at baseline.

\section{Feasibility and Acceptability of ARTEMIS+CM}

The 12 participants randomized to receive the ARTEMIS completed $98 \%$ (59 of 60) scheduled sessions during the eight remaining weeks of CM. On average, participants receiving ARTEMIS reported a marginally significant increase in positive affect across the five-session intervention from a $32.5(\mathrm{SD}=7.3)$ at session $1-35.0(\mathrm{SD}=8.2)$ at session $5(B$ $=2.69, p=.052$; Cohen's $d=0.32)$. There were no significant changes in negative affect from 16.0 $(\mathrm{SD}=7.4)$ at session $1-14.8(\mathrm{SD}=8.9)$ at session $5(\mathrm{~B}=-0.82, p=.70$; Cohen's $d=-0.15)$.

There were no significant differences in urine toxicology results during $\mathrm{CM}$ as a function of intervention assignment. Overall, participants provided an average of $30.5(\mathrm{SD}=6.3)$ urine samples that were non-reactive for methamphetamine out of 36 possible ( $85 \%)$. Participants provided an average of $20.7(\mathrm{SD}=10.7)$ urine samples in a row that were non-reactive for methamphetamine, which is equivalent to approximately seven weeks of continuous abstinence during CM.

Of the 11 ARTEMIS+CM participants who completed the 2-month follow-up assessment, the average response for recommending ARTEMIS to a friend who uses methamphetamine was 8.7 ( $\mathrm{SD}=2.2$ ). During exit interviews, participants noted personal benefits derived from the ARTEMIS intervention.

It really clicked, and I was like, 'Wow! This is pleasurable!' and that almost changed like, my whole recovery experience. In the past, in rehabs, it was like, 'You're a bad person.' And this changed it, for some reason. I was able to have like, a new experience with recovery. (Caucasian, HIV-positive participant)

Since I've been doing this study, I've been using my brain and thinking my way out of situations. Thinking my way on controlling my stress level and controlling my anger, taking a deep breath... actually I'm a better man today. I'm more stronger, in street terms I have more game since you guys. (African American, HIV-negative participant)

Now I pay attention to my emotions, my body, my breathing, and the person who I am. I started attending 12-step groups because this intervention made me want to become a better person, a better friend, a better partner. (Hispanic/Latino, HIVpositive participant)

This [intervention] made me more aware that I had a problem, and that it's not just a meth problem; it's an alcohol problem. So, I've started working on some stuff, and I set some goals. This experience steered me the right way. (Caucasian, HIVnegative participant) 


\section{Preliminary Outcomes of ARTEMIS+CM}

As shown in Table 2, participants in ARTEMIS+CM reported modest increases in positive affect at two-month post-randomization $(B=4.67, p<.05$; Cohen's $d=0.24)$, but these were not maintained through the 6-month follow-up. In contrast, participants in CM-only reported significant reductions in negative affect immediately at two-month post-randomization $(B=$ $-7.69, p<.05$; Cohen's $d=-0.23$ ) that were not maintained through the 6-month follow-up. No other significant changes in any outcome were observed over the 6-month follow-up.

\section{Discussion}

This formative clinical research pilot tested a positive affect intervention that is designed to boost and extend the effectiveness of CM by promoting resilience among methamphetamine-usingMSM.Findingssupportthefeasibilityandacceptabilityof this5sessionpositiveaffectinterventiondeliveredconcurrently with CM. Participants completed almost all of the scheduled ARTEMIS intervention sessions as well as noted ways in which the positive affect skills enhanced self-awareness and supported the process of recovery.

The most appropriate primary objectives of a pilot RCT are to examine feasibility and acceptability, and there is increasing recognition that a phase II efficacy RCT is necessary to reliably estimate the true direction and magnitude of the effects for a novel intervention (Kraemer, Mintz, Noda, Tinklenberg, \& Yesavage, 2006). Although effect size estimates from pilot studies should be interpreted with caution, participants randomized to receive ARTEMIS+CM reported short-term increases in positive affect that were not sustained over the 6-month follow-up. Consistent with prior RCTs of stress and coping interventions for HIV-positive persons (Antoni et al., 2006; Carrico et al., 2009; Morin et al., 2008), even short-term increases in positive affect may have important implications for reductions in stimulant use and HIV-related health behavior change (Carrico et al., 2010; Carrico \& Moskowitz, 2014; Mustanski, 2007).

In addition to the small sample size, there were three other major limitations to this pilot RCT. First, participants were enrolled based on self-reported methamphetamine use. Although participants completed a detailed screening process for the CM program and the present pilot RCT, it is plausible that some over-reported methamphetamine use to receive the incentives provided. Second, urine samples were only screened for methamphetamine during CM visits and participants who used other stimulants (e.g., cocaine) still received the incentives as positive reinforcement for abstinence. Third, participants in CM-only received treatment as usual without an attempt to match for facilitator contact time or study incentives that were provided to those randomized to receive ARTEMIS+CM(i.e., cash, an iPod shuffle). Our ongoing phase II RCT of ARTEMIS+CM addresses each of these limitations by enrolling only those with biologically confirmed recent methamphetamine use, distributing CM incentives only for urine screens that are negative for methamphetamine and cocaine metabolites, and providing control participants with identical incentives and comparable contact time with study staff (NCT01926184). Future research should also measure pre-exposure prophylaxis utilization and HIV viral suppression as important biomedical interventions that may mitigate risk of HIV seroconversion or onward transmission in this population. 
Taken together, findings from this study indicate that a positive affect intervention delivered during CM with methamphetamine-using MSM is feasible and acceptable. Short-term increases in positive affect following ARTEMIS+CM also provide some of the first evidence that methamphetamine-using MSM may be responsive to interventions designed to build resilience. More definitive clinical research is warranted to examine the efficacy of ARTEMIS+CM with methamphetamine-using MSM.

\section{Acknowledgments}

This work was supported as a Pilot Project of the University of California, San Francisco Center for AIDS Prevention Studies, which was funded by the National Institute of Mental Health (P30MH062246; Lightfoot, PI). Additional support was provided by the National Institute of Mental Health (K24-MH093225; Moskowitz, PI) and the National Institute on Drug Abuse (R01-DA033854; Carrico \& Moskowitz, PIs).

\section{References}

Antoni MH, Carrico AW, Duran RE, Spitzer S, Penedo F, Ironson G, Schneiderman N. Randomized clinical trial of cognitive behavioral stress management on human immunodeficiency virus viral load in gay men treated with highly active antiretroviral therapy. Psychosomatic Medicine. 2006; 68(1):143-151.10.1097/01.psy.0000195749.60049.63 [PubMed: 16449425]

Bowen S, Chawla N, Collins SE, Witkiewitz K, Hsu S, Grow J, Marlatt A. Mindfulness-based relapse prevention for substance use disorders: A pilot efficacy trial. Substance Abuse. 2009; 30(4):295305.10.1080/08897070903250084 [PubMed: 19904665]

Bowen S, Witkiewitz K, Clifasefi SL, Grow J, Chawla N, Hsu SH, Larimer ME. Relative efficacy of mindfulness-based relapse prevention, standard relapse prevention, and treatment as usual for substance use disorders: A randomized clinical trial. JAMA Psychiatry. 2014; 71(5):547556.10.1001/jamapsychiatry.2013.4546 [PubMed: 24647726]

Carrico, AW. Positive emotion: The sirens' song of substance use and the Trojan horse of recovery from addiction. In: Gruber, J.; Moskowitz, JT., editors. The dark and light sides of positive emotion. Oxford; Oxford University Press; 2014. p. 415-469.

Carrico AW, Chesney MA, Johnson MO, Morin SF, Neilands TB, Remien RH, NIMH Healthy Living Project Team. Randomized controlled trial of a cognitive-behavioral intervention for HIV-positive persons: An investigation of treatment effects on psychosocial adjustment. AIDS and Behavior. 2009; 13(3):555-563.10.1007/s10461-008-9429-6 [PubMed: 18626764]

Carrico AW, Flentje A, Gruber VA, Woods WJ, Discepola MV, Dilworth SE, Siever MD. Community-based harm reduction substance abuse treatment with methamphetamine-using men who have sex with men. The Journal of Urban Health. 201410.1007/s11524-014-9870-y

Carrico AW, Johnson MO, Colfax GN, Moskowitz JT. Affective correlates of stimulant use and adherence to anti-retroviral therapy among HIV-positive methamphetamine users. AIDS and Behavior. 2010; 14(4):769-777.10.1007/s10461-008-9513-y [PubMed: 19125321]

Carrico AW, Moskowitz JT. Positive affect promotes engagement in care after HIV diagnosis. Health Psychology. 2014; 33(7):686-689.10.1037/hea0000011 [PubMed: 24245846]

Carrico AW, Riley ED, Johnson MO, Charlebois ED, Neilands TB, Remien RH, Chesney MA. Psychiatric risk factors for HIV disease progression: The role of inconsistent patterns of antiretroviral therapy utilization. Journal of Acquired Immune Deficiency Syndromes. 2011; 56(2): 146-150.10.1097/QAI.0b013e318201df63 [PubMed: 21116186]

Carrico AW, Woods WJ, Siever MD, Discepola MV, Dilworth SE, Neilands TB, Moskowitz JT. Positive affect and processes of recovery among treatment-seeking methamphetamine users. Drug and Alcohol Dependence. 2013; 132(3):624-629.10.1016/j.drugalcdep.2013.04.018 [PubMed: 23684632]

Des Jarlais DC, Paone D, Milliken J, Turner CF, Miller H, Gribble J, Friedman SR. Audio-computer interviewing to measure risk behaviour for HIV among injecting drug users: A quasi-randomised trial. Lancet. 1999; 353(9165):1657-1661. [PubMed: 10335785] 
Folkman S, Moskowitz JT. Positive affect and the other side of coping. American Psychologist. 2000; 55(6):647-654. [PubMed: 10892207]

Fredrickson BL. What good are positive emotions? Review of General Psychology. 1998; 2(3):300319.10.1037/1089-2680.2.3.300 [PubMed: 21850154]

Fredrickson BL, Tugade MM, Waugh CE, Larkin GR. What good are positive emotions in crises? A prospective study of resilience and emotions following the terrorist attacks on the United States on September 11th, 2001. Journal of Personality and Social Psychlogy. 2003; 84:365-376.

Herrick AL, Lim SH, Plankey MW, Chmiel JS, Guadamuz TE, Kao U, Stall R. Adversityand syndemic production among men participating in the multicenter AIDS cohort study: A life-course approach. American Journal of Public Health. 2013; 103(1):79-85.10.2105/AJPH.2012.300810 [PubMed: 23153154]

Herrick AL, Stall R, Goldhammer H, Egan JE, Mayer KH. Resilience as a research framework and as a cornerstone of prevention research for gay and bisexual men: Theory and evidence. AIDS and Behavior. 2014; 18(1):1-9.10.1007/s10461-012-0384-x [PubMed: 23321946]

Izard, CE. Human emotions. New York: Plenum; 1977.

Johnson MO, Carrico AW, Chesney MA, Morin SF. Internalized heterosexism among HIV-positive, gay-identified men: Implications for HIV prevention and care. Journal of Consulting and Clinical Psychology. 2008; 76(5):829-839.10.1037/0022-006X.76.5.829 [PubMed: 18837600]

Koblin BA, Husnik MJ, Colfax G, Huang Y, Madison M, Mayer K, Buchbinder S. Risk factors for HIV infection among men who have sex with men. AIDS. 2006; 20(5):731-739.10.1097/01.aids. 0000216374.61442.55 [PubMed: 16514304]

Kraemer HC, Mintz J, Noda A, Tinklenberg J, Yesavage JA. Caution regarding the use of pilot studies to guide power calculations for study proposals. Archives of General Psychiatry. 2006; 63(5):484489.10.1001/archpsyc.63.5.484 [PubMed: 16651505]

Ma Y, Mazumdar M, Memtsoudis SG. Beyond repeated-measures analysis of variance: Advanced statistical methods for the analysis of longitudinal data in anesthesia research. Regional Anesthesia and Pain Medicine. 2012; 37(1):99-105.10.1097/AAP.0b013e31823ebc74 [PubMed: 22189576]

Mayer KH, Skeer MR, O'Cleirigh C, Goshe BM, Safren SA. Factors associated with amplified HIV transmission behavior among American men who have sex with men engaged in care:

Implications for clinical providers. Annals of Behavioral Medicine. 2014; 47(2):165-171.10.1007/ s12160-013-9527-1 [PubMed: 23873338]

Menza TW, Jameson DR, Hughes JP, Colfax GN, Shoptaw S, Golden MR. Contingency management to reduce methamphetamine use and sexual risk among men who have sex with men: A randomized controlled trial. BMC Public Health. 2010; 10:774.10.1186/1471-2458-10-774 [PubMed: 21172026]

Morin SF, Shade SB, Steward WT, Carrico AW, Remien RH, Rotheram-Borus MJ, Healthy Living Project Team. A behavioral intervention reduces HIV transmission risk by promoting sustained serosorting practices among HIV-infected men who have sex with men. Journal of Acquired Immune Deficiency Syndromes. 2008; 49(5):544-551.10.1097/QAI.0b013e31818d5def [PubMed: 18989221]

Moskowitz JT, Hult JR, Duncan LG, Cohn MA, Maurer S, Bussolari C, Acree M. Apositiveaffectinterventionfor people experiencing health-related stress: Development and nonrandomized pilot test. Journal of Health Psychology. 2012; 17(5):676-

692.10.1177/1359105311425275 [PubMed: 22021272]

Mustanski B. The influence of state and trait affect on HIV risk behaviors: A daily diary study of MSM. Health Psychology. 2007; 26(5):618-626.10.1037/0278-6133.26.5.618 [PubMed: 17845113]

Mustanski B, Garofalo R, Herrick A, Donenberg G. Psychosocial health problems increase risk for HIV among urban young men who have sex with men: Preliminary evidence of a syndemic in need of attention. Annals of Behavioral Medicine. 2007; 34(1):3745.10.1080/08836610701495268 [PubMed: 17688395]

Ostrow DG, Plankey MW, Cox C, Li X, Shoptaw S, Jacobson LP, Stall RC. Specific sex drug combinations contribute to the majority of recent HIV seroconversions among MSM in the 
MACS. Journal of Acquired Immune Deficiency Syndromes. 2009; 51(3):349-355.10.1097/QAI. 0b013e3181a24b20 [PubMed: 19387357]

Parsons JT, Grov C, Golub SA. Sexual compulsivity, co-occurring psychosocial health problems, and HIV risk among gay and bisexual men: Further evidence of a syndemic. American Journal of Public Health. 2012; 102(1):156-162.10.2105/AJPH.2011.300284 [PubMed: 22095358]

Prendergast M, Podus D, Finney J, Greenwell L, Roll J. Contingency management for treatment of substance use disorders: A meta-analysis. Addiction. 2006; 101(11):1546-1560. [PubMed: 17034434]

Reback CJ, Peck JA, Dierst-Davies R, Nuno M, Kamien JB, Amass L. Contingency management among homeless, out-of-treatment men who have sex with men. Journal of Substance Abuse Treatment. 2010; 39(3):255-263.10.1016/j.jsat.2010.06.007 [PubMed: 20667681]

Shoptaw S, Klausner JD, Reback CJ, Tierney S, Stansell J, Hare CB, Dang J. A public health response to the methamphetamine epidemic: The implementation of contingency management to treat methamphetamine dependence. BMC Public Health. 2006; 6:214.10.1186/1471-2458-6-214 [PubMed: 16919170]

Shoptaw S, Reback CJ, Peck JA, Yang X, Rotheram-Fuller E, Larkins S, Hucks-Ortiz C. Behavioral treatment approaches for methamphetamine dependence and HIV-related sexual risk behaviors among urban gay and bisexual men. Drug and Alcohol Dependence. 2005; 78(2):125134.10.1016/j.drugalcdep.2004.10.004 [PubMed: 15845315]

Stall, R.; Friedman, M.; Catania, JA. Interacting epidemics and gay men's health: A theory of syndemic production among urban gay men. In: Wolitiski, RJ.; Stall, R.; Valdiserri, RO., editors. Unequal opportunity: Health disparities affecting gay and bisexual men in the United States. New York: Oxford University Press; 2008. p. 251-274.

Stall R, Mills TC, Williamson J, Hart T, Greenwood G, Paul J, Catania JA. Association of cooccurring psychosocial health problems and increased vulnerability to HIV/AIDS among urban men who have sex with men. Am J Public Health. 2003; 93(6):939-942. [PubMed: 12773359]

Strona FV, McCright J, Hjord H, Ahrens K, Tierney S, Shoptaw S, Klausner JD. The acceptability and feasibility of the Positive Reinforcement Opportunity Project, a community-based contingency management methamphetamine treatment program for gay and bisexual men in San Francisco. Journal of Psychoactive Drugs. 2006; (Suppl 3):377-383. [PubMed: 17357529]

Thomas DR. Ageneralinductiveapproachforanalyzingqualitative evaluation data. American Journal of Evaluation. 2006; 27:237-246.

Witkiewitz K, Bowen S, Douglas H, Hsu SH. Mindfulness-based relapse prevention for substance craving. Addictive Behaviors. 2013; 38(2):1563-1571.10.1016/j.addbeh.2012.04.001 [PubMed: 22534451] 


\section{Table 1}

Positive affect intervention protocol for methamphetamine-using MSM

\begin{tabular}{|c|c|c|}
\hline Session & Positive affect regulation skills & Adapted intervention content \\
\hline 1 & $\begin{array}{l}\text { Noticing positive events } \\
\text { Capitalizing on positive events } \\
\text { Gratitude }\end{array}$ & $\begin{array}{l}\text { Psychoeducation on stimulant withdrawal } \\
\text { Capitalizing on non-reactive urine toxicology screens } \\
\text { Breathing retraining }\end{array}$ \\
\hline 2 & Mindfulness (informal and formal) & Breath meditation \\
\hline 3 & Positive reappraisal & $\begin{array}{l}\text { Problem-focused coping and reasoned action } \\
\text { Mountain meditation }\end{array}$ \\
\hline 4 & $\begin{array}{l}\text { Strengths } \\
\text { Attainable goals }\end{array}$ & \\
\hline 5 & Altruism & Loving-kindness meditation \\
\hline
\end{tabular}


Table 2

Preliminary outcomes of the pilot randomized controlled trial of ARTEMIS+CM $(N=21)$

\begin{tabular}{|c|c|c|}
\hline & $\begin{array}{l}\text { ARTEMIS+CM } \\
(N=12) \\
M \text { (SD) }\end{array}$ & $\begin{array}{l}\text { CM-only } \\
(N=9) \\
M(S D)\end{array}$ \\
\hline \multicolumn{3}{|l|}{ Positive affect } \\
\hline Baseline & $30.3(9.9)$ & $30.3(7.3)$ \\
\hline 2 Months & $34.8(9.2)^{*}$ & $32.8(7.7)$ \\
\hline 3 Months & $34.1(7.4)$ & $35.4(8.1)$ \\
\hline 6 Months & $32.0(7.9)$ & $33.3(8.9)$ \\
\hline \multicolumn{3}{|c|}{ Negative affect } \\
\hline Baseline & $17.7(6.2)$ & $20.8(8.3)$ \\
\hline 2 Months & $14.8(9.1)$ & $12.8(8.6)^{*}$ \\
\hline 3 Months & $16.5(7.5)$ & $15.0(4.5)$ \\
\hline 6 Months & $17.3(8.8)$ & $15.1(8.8)$ \\
\hline \multicolumn{3}{|c|}{ Total number of risky anal sex partners } \\
\hline Baseline & $0.1(0.3)$ & $0.2(0.7)$ \\
\hline 3 Months & $0.2(0.4)$ & $1.3(3.5)$ \\
\hline 6 Months & $0.0(0.0)$ & $0.0(0.0)$ \\
\hline \multicolumn{3}{|c|}{ Number of risky anal sex partners on methamphetamine } \\
\hline Baseline & $0.0(0.0)$ & $0.2(0.7)$ \\
\hline 3 Months & $0.1(0.3)$ & $0.0(0.0)$ \\
\hline 6 Months & $0.0(0.0)$ & $0.0(0.0)$ \\
\hline \multicolumn{3}{|c|}{ Self-reported methamphetamine use (past 30 days) } \\
\hline Baseline & $1.3(1.7)$ & $1.3(3.0)$ \\
\hline 2 Months & $2.4(3.3)$ & $2.3(5.2)$ \\
\hline 3 Months & $4.4(7.5)$ & $0.1(0.4)$ \\
\hline \multirow[t]{2}{*}{6 Months } & $4.3(6.8)$ & $0.1(0.4)$ \\
\hline & $N(\%)$ & $N(\%)$ \\
\hline \multicolumn{3}{|c|}{ Reactive urine sample for stimulants } \\
\hline Baseline & $1(8.3)$ & $1(12.5)$ \\
\hline 2 Months & $2(22.2)$ & $1(12.5)$ \\
\hline 3 Months & $3(27.3)$ & $0(0.0)$ \\
\hline 6 Months & $2(20.0)$ & $0(0.0)$ \\
\hline
\end{tabular}

